\title{
COMMENT
}

\section{The European Medicines Agency's goals for regulatory science to 2025}

\author{
Philip A. Hines ${ }^{1,2,3 *}$, Richard H. Guy ${ }^{1,4,5}$, Anthony J. Humphreys ${ }^{1}$ and \\ Marisa Papaluca-Amati ${ }^{1}$
}

It is increasingly important that regulatory agencies catalyse the translation of scientific and technological innovation into improved patient-centred health care. Here, we highlight the strategic goals of the European Medicines Agency to advance regulatory science and optimize the opportunities arising in areas such as cell and gene therapies, big data and artificial intelligence.

Transformational advances in cell or gene-based therapies, drug-device combinations, novel clinical trial design, real-world evidence, 'big data' and artificial intelligence (AI) present an important challenge to regulatory scientists. Consequently, in 2016, the European Medicines Agency (EMA) formalized a Regulatory Science Observatory to monitor emerging trends in science and technology, and to direct the use of resources and external collaborations to advance regulatory science. The ambition was to identify priority areas where new or enhanced engagement is essential to the continued success of the EMA's vision - to catalyse and enable science to be translated into patient-centred care and its delivery in evolving health-care systems (see Related links). To fulfil this objective, trends in science, technology and regulatory tools affecting EMA operations were surveyed in-depth and the results were supplemented with outreach activities with stakeholders at all levels of the medicine development pathway (see Supplementary information). The culmination of this effort was a workshop held at the EMA in October 2018, at which five strategic goals were outlined (see Supplementary information and Related links). These goals are highlighted here.

\section{Strategic goals}

Catalysing the integration of science and technology in medicines development. The specific aim is to encourage and enable the latest scientific and technological knowledge to be built into medicines development. This requires an ongoing dialogue between regulators, academic research centres and developers at all stages of a product's life cycle. Specific recommendations include:

- Enhance early engagement with novel biomarker developers to facilitate regulatory qualification.

- Support evidence generation for advanced therapy medicinal products (ATMPs). The EMA proposes to increase early interaction between developers and regulators, as well as health technology assessment bodies (HTAs) and payers, to ensure that regulatory concerns for ATMPs are addressed as early as possible and to facilitate faster access for patients.

- Identify bottlenecks and propose modernization of relevant regulations to facilitate manufacturing of novel products. Advances in continuous, additive and point-of-care manufacturing fit poorly into traditional regulatory models, and may require adaptation of good manufacturing practice (GMP) requirements and standards and the development of specific regulatory guidance and monitoring.

- Create an integrated evaluation pathway for the assessment of medical devices, in vitro diagnostics and 'borderline' products. Increasingly, complex products are emerging that combine a medicine and a medical device with a mixed mechanism of action. Innovative medicines may also depend on the use of associated in vitro diagnostics. To support development and regulation of such borderline products, the EMA requires integrated competence and expertise, and collaboration with bodies responsible for regulating medical devices.

- Create complementary and flexible advice mechanisms to support innovative product development.

Driving collaborative evidence generation and improving the scientific quality of evaluations. The goal is to provide regulators with better evidence to underpin regulatory assessment. New digital tools mean that data can be more widely and efficiently collected throughout the life cycle of a medicine, from preclinical development, through the clinical trial process and into real-world use. This is particularly important for special populations (for example, children, patients with rare diseases or those with conditions that have a high individual and/or public health burden), and it offers an opportunity to better capture patient preferences during the evaluation process and make clinical 
development and regulation more cost effective. Specific recommendations include:

- Drive adoption of novel clinical trial practices that facilitate clinical trial authorization, good clinical practice and HTA acceptance.

- Establish a dedicated AI test 'laboratory' to explore the application of innovative digital technology to support data-driven decisions.

- Develop the capability to assess complex data sets captured by technology such as wearables.

- Develop the capability to analyse individual patient data to support decision making.

Advancing patient-centred access to medicines in partnership with health-care systems. This objective aims to ensure that patients receive timely access to affordable, high-quality medicines and that health-care stakeholders have the information needed to guide correct prescription and use. Specific recommendations include:

- Co-develop core health-related quality-of-life patient-reported outcomes (PROs) with HTAs to implement in trials and to bridge the gap with comparative effectiveness assessment. It is increasingly accepted that crucial insight can come from a more holistic assessment of the impact of a medicine on the patient's quality of life.

- Enable information exchange with HTAs to support bridging from benefit-risk to relative effectiveness assessment. New standards and guidelines should be developed between stakeholders to avoid divergences in evidential standards.

- Create a sustainable, quality-assured, flexible framework delivering rapid access to and analysis of representative, longitudinal real-world data throughout a product's life cycle. The often heterogeneous nature of sources for such data requires development of improved analytical and epidemiological methodologies to deliver robust evidence.

- Enable involvement of payers' requirements in the prospective discussion of evidence-generation plans.

Addressing emerging health threats and availability/ therapeutic challenges. This goal aims to ensure that the EMA can respond effectively to address the need for, and availability of, medicinal products to tackle existing and emerging health threats (such as the need for new antimicrobials and methods to manage antimicrobial resistance, and initiatives to improve communication and build public understanding and trust in vaccines). Another area to be addressed is the uneven availability of medicines in the European Union, either because they are not marketed or due to supply disruptions. Specific recommendations include:

- Implement the EMA's health threats plan, ring-fence resources and refine preparedness approaches.

- Evolve regulatory guidance and support alternative approaches to new antibacterial drug development and to innovative methods to prevent and treat infections.

- Explore mechanisms to increase manufacturing capacity in Europe and internationally.
- Advance methods and tools such as biomarkers to characterize immune responses and to support definition of vaccine quality attributes.

- Communicate proactively with stakeholders on benefit-risk profiles of vaccines using evidence-based tools to tackle vaccine hesitancy.

- Support the development and implementation of a drug repurposing framework, which has the potential to reduce the time and expense of development and offer additional therapeutic options to patients.

Enabling and leveraging research and innovation in regulatory science. It is envisaged that this aim, which is the key to delivery of the four strategic goals described above, can be achieved by establishing a novel regulatory science and innovation platform in partnership with academic research centres (see Related links). This will provide a mechanism for scientists in the EMA regulatory network and academia to collaborate in identifying and tackling fundamental research questions of high relevance, such as PROs, omics-based diagnostics, drugdevice combinations, modelling and simulation, big data and AI. It will also enable the development of new regulatory competency in emerging areas of innovation, such as precision medicine and wearables. In addition, realization of this strategic objective will improve access to expertise in regulatory science to better track innovation and enable enhanced insight into the development of novel medicinal products.

\section{Next steps}

It is envisaged that the core recommendations will be realized by series of actions that identify the practical steps to be undertaken (detailed in Supplementary information). A draft document, 'Regulatory Science to 2025, presents in detail the proposed strategy outlined here and is available for public consultation during the first half of 2019 (see Related links). Interested stakeholders are strongly encouraged to read the consultation document and to provide comments on the strategy proposed. Following consideration of the feedback received, a final version of the document will be released and presented publicly in the fourth quarter of 2019.

Acknowledgements

The authors thank colleagues and stakeholders for input in shaping the strategy.

Competing interests

The authors declare no competing interests.

Disclaimer

The views expressed in this article are the personal views of the author(s) and may not be understood or quoted as being made on behalf of or reflecting the position of the European Medicines Agency or one of its committees or working parties.

\section{RELATED LINKS}

EU's Innovative Medical Technology and EMA's Measures:

https://www.pmda.go.jp/files/000220949.pdf

Multistakeholder workshop to launch consultation on European Medicines Agency (EMA) human regulatory science to 2025: https://www.ema.europa. eu/en/events/multistakeholder-workshop-launch-consultation-europeanmedicines-agency-ema-human-regulatory-science Regulatory science to 2025: https://www.ema.europa.eu/en/about-us/ how-we-work/regulatory-science-2025 